\title{
Seleção genética em teste de progênies de irmãos completos de Eucalyptus para a produção de carvão vegetal
}

\author{
Genetic selection in Eucalyptus full-sibs \\ families for charcoal production
}

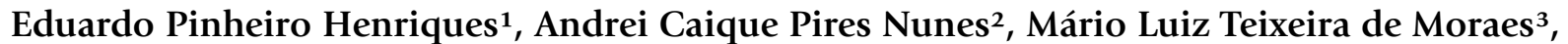
Marcos Deon Vilela de Resende ${ }^{4}$, Alexandre Magno Sebbenn ${ }^{5}$ e Marcela Aparecida de Moraes ${ }^{6}$
\end{abstract}

\begin{abstract}
Resumo
O objetivo deste estudo foi avaliar um teste de progênies de irmãos completos de Eucalyptus aos 2, 5 e 7 anos de idade para selecionar genitores e indivíduos superiores em um programa de seleção recorrente recíproca no estado de Minas Gerais. O delineamento experimental utilizado foi de blocos ao acaso com 286 famílias de irmãos completos, oito repetições e parcelas lineares de seis plantas. Os caracteres avaliados foram a altura (ALT), diâmetro a altura do peito (DAP) e volume (VOL). A herdabilidade individual no sentido restrito $\left(h^{2}\right)$ para VOL para todas as idades foi de aproximadamente o dobro da herdabilidade dos efeitos de dominância da população $\left(h_{\text {dom }}^{2}\right)$. A herdabilidade individual no sentido amplo $\left(H^{2}\right)$ para o VOL foi média (variou entre idades de 0,28 a 0,39 ), o que indica a possibilidade de ganhos genéticos com a seleção dentro da população. Foram selecionados 256 indivíduos de 32 famílias de maior VOL (média de $74,24 \mathrm{~m}^{3}$.ha- ${ }^{-1}$.ano-1) para formação do pomar de recombinação e 65 indivíduos para estabelecimento do pomar de hibridação. Foram também selecionados os dez indivíduos com maior VOL para clonagem (média de $86,08 \mathrm{~m}^{3} \cdot \mathrm{ha}^{-1}$.ano-1). A metodologia de seleção genética utilizada possibilitou o estudo quantitativo da população de irmãos completos e direcionamento de cruzamentos futuros para o programa de seleção recorrente recíproca em Minas Gerais.
\end{abstract}

Palavras-chave: Parâmetros genéticos; Seleção Recorrente Recíproca; Teste de progênies.

\begin{abstract}
The objective of this study was to evaluate a progeny test of Eucalyptus full-sib families at 2, 5 and 7 years of age to select parents and superior individuals in a reciprocal recurrent selection program in the state of Minas Gerais. The experimental design was a randomized complete block design with 286 full-sib families, eight replications and linear plots of six plants. The evaluated traits were height (ALT), diameter at breast height (DAP) and volume (VOL). The individual heritability in strict sense $\left(h_{a}^{2}\right)$ for VOL and for all ages was approximately double the heritability of the dominance effects of the population $\left(h_{\text {diom }}^{2}\right)$. The individual heritability in the broad sense $\left(H_{a}^{2}\right)$ for the VOL was medium (ranged between ages from 0.28 to 0.39 ), which indicates the possibility of genetic gains with selection within the population. A total of 256 individuals were selected from 32 families with a highest VOL (mean of $74.24 \mathrm{~m}^{3}$.ha-1.. $\mathrm{year}^{-1}$ ) to establish a seed orchard and 65 progenies were selected for a hybrid seed orchard. Ten individuals with highest VOL were also selected (mean of $86.08 \mathrm{~m}^{3} \cdot \mathrm{ha}^{-1}$.year ${ }^{-1}$ ). The genetic selection methodology used allowed the quantitative study of the full-sib population and the direction of future crosses for the reciprocal recurrent selection program in Minas Gerais.
\end{abstract}

Keywords: Genetic parameters; Progeny test; Reciprocal Recurring Selection.

${ }^{1}$ Doutor em Engenharia Florestal. UNESP - Universidade Estadual Paulista "Júlio de Mesquita Filho" / FCA - Faculdade de Ciências Agronômicas. Caixa Postal 237 - 18610-237 - Botucatu, SP, Brasil. E-mail: eduardopinheirohenriques@gmail.com.

2Professor Adjunto de Melhoramento Florestal e Biotecnologia. UFSB - Universidade Federal do Sul da Bahia. Campus Jorge Amado - Rodovia de Acesso para Itabuna, Km 39 - 45613-204 - Itabuna, BA, Brasil. E-mail: andreicaique@gmail.com. 3Professor Titular do Departamento de Fitotecnia e Tecnologia de Alimentos e Sócio Econômica. UNESP - Universidade Estadual Paulista "Júlio de Mesquita Filho" / FEIS- Faculdade de Engenharia de Ilha Solteira. Avenida Brasil, n. 56 - Centro - 15385-000 - Ilha Solteira, SP, Brasil. E-mail: teixeira@agr.feis.unesp.br.

${ }^{4}$ Pesquisador Doutor. Embrapa - Empresa Brasileira de Pesquisa Agropecuária / Centro Nacional de Florestas. Estrada da Ribeira, Km11 - 83411 -000 - Colombo, PR, Brasil. E-mail: marcos.deon@gmail.com.

${ }^{5}$ Pesquisador Doutor. IF - Instituto Florestal de São Paulo. Estação Experimental de Tupi. Rodovia Luiz de Queiroz, KM 149,5 - Tupi - 13400-970 - Piracicaba, SP, Brasil. E-mail: alexandresebbenn@yahoo.com.br.

${ }^{6}$ Pós Doutoranda em Fisiologia Vegetal. USP - Universidade de São Paulo / FFCLRP - Faculdade de Filosofia, Ciências e Letras de Ribeirão Preto. Av. Bandeirantes, 3.900 - Vila Monte Alegre - 14040-900 - Ribeirão Preto, SP. Brasil. E-mail: marcela.apmoraes@gmail.com.

Sci. For., Piracicaba, v. 46, n. 119, p. 405-413, set. 2018 DOI: dx.doi.org/10.18671/scifor.v46n119.08 
Henriques et al. - Seleção genética em teste de progênies de irmãos

completos de Eucalyptus para a produção de carvão vegetal

\section{INTRODUÇÃO}

Em 2015 a área ocupada por plantio de Eucalyptus, Pinus e outras espécies (entre elas Seringueira, Acácia, Teca e Paricá) no Brasil foi de 7.801.047 ha, sendo 5.630.607 ha (72\%) de Eucalyptus, 1.581.239 ha (20\%) de Pinus e 589.201 ha (8\%) de outras espécies, conforme Indústria Brasileira de Árvores (IBÁ, 2016). Os Estados de Minas Gerais, São Paulo, Mato Grosso do Sul e Bahia se destacaram no cenário nacional como detentores de $68 \%$ da área total dos plantios de Eucalyptus.

A partir da metade da década de 1970, Empresas integradas/verticalizadas, (de celulose e siderúrgicas entre as demais) que plantavam florestas para consumo próprio e outras, com o objetivo de formar patrimônio florestal para negociação futura, utilizaram intensivamente de incentivos fiscais para reflorestamentos com espécies exóticas. A falta de dados e de tecnologia florestais forçou o seguimento florestal a utilizar técnicas agrícolas para a implantação dos plantios. A introdução das espécies foi baseada nas recomendações de Golfari (1975), que, por exemplo, indicava para plantios especialmente na região do cerrado de Minas Gerais as espécies: E. camaldulensis, E. tereticornis, E. grandis e E. saligna. Florestas nativas de cerrado que nesta região tinham baixa produtividade em termos de volume de madeira $\left(1,5 \mathrm{~m}^{3} \cdot \mathrm{ha}^{-1}\right.$.ano $\left.{ }^{-1}\right)$ foram substituídas por espécies do gênero Eucalyptus introduzidas, as quais eram mais produtivas $\left(9,0 \mathrm{~m}^{3} \cdot \mathrm{ha}^{-1} \cdot \mathrm{ano}^{-1}\right)$. No caso das indústrias siderúrgicas, o carvão vegetal produzido de floresta plantada foi de grande relevância, por diminuir a produção de carvão a partir da floresta nativa, bem como reduziu a importação do coque mineral, que apresentava o agravante de ser altamente poluente. A baixa produtividade levou também as empresas a imobilizarem um grande patrimônio fundiário, o que agravou os conflitos de terra em diversas regiões do país, notadamente nas extensas áreas mais pobres, e sem recursos como o Vale do Jequitinhonha em Minas Gerais e a iniciarem programas de melhoramento genético sólido para aumentar a produtividade dos plantios de Eucalyptus.

Programas de melhoramento genético de espécies de Eucalytus possibilitaram aumento na produtividade dos plantios, pela definição de espécies e procedências mais adaptadas aos ambientes de cultivo, adequação ao produto final, métodos de propagação, hibridação e silvicultura clonal, devido à possibilidade do planejamento dos cruzamentos pela indução floral em genitores enxertados e conduzidos em casa de vegetação. Essas técnicas foram determinantes para o sucesso do programa de melhoramento estabelecer estratégias que tenham como fundamento a condução das hibridações, visando ao melhoramento do próprio híbrido ao longo das gerações com aumento contínuo da heterose. A expectativa é de que os híbridos gerados entre os genitores com base em seus valores genéticos preditos em populações divergentes e, portanto, portadores de boa capacidade geral e específica de combinação apresentem superioridade ao longo das gerações em esquemas de seleção recorrente recíproca (ROCHA et al., 2007).

As estratégias ideais de melhoramento de híbridos de Eucalipto com vistas à clonagem devem basear-se em seleção recorrente recíproca (SSR), conforme sugerido por Resende e Higa (1990). Esse método tem sido usado por várias indústrias de celulose no Brasil, África do Sul e Congo, geralmente envolvendo o híbrido entre as espécies Eucalyptus urophylla x Eucalyptus grandis. Por examplo, o programa de SRR da V\&M Florestal iniciou-se em 1980, com as espécies Eucalyptus camaldulensis e E. urophylla, com produtividade em termos de volume de madeira de $4 \mathrm{~m}^{3}$.ha ${ }^{-1}$.ano ${ }^{-1}$ e resultaram na produtividade média de $36 \mathrm{~m}^{3} \cdot \mathrm{ha}^{-1} \cdot \mathrm{ano}^{-1}$, o que corresponde a ganhos genéticos de $900 \%$ (ASSIS, 1996). Este aumento expressivo nos ganhos de produtividade pode ser atribuído, principalmente à hibridação interespecífica (ASSIS et al., 2007). Esta técnica apresenta a introgressão de genes exógenos de interesse, por polinização controlada ou aberta, de pólen de espécies com as características desejadas (BOUDET, 1998).

Todavia, o êxito do melhoramento genético está associado à capacidade de acerto na escolha dos melhores genitores para serem os pais das próximas gerações melhoradas (CRUZ; CARNEIRO, 2003). Para a identificação dos indivíduos portadores de genes desejáveis é necessária a avaliação genética dos candidatos à seleção. Em plantas perenes como Eucalyptus, recomenda-se que a seleção seja feita com base nos valores genéticos aditivos dos indivíduos que serão utilizados na recombinação e nos valores genotípicos dos indivíduos que serão clonados (RESENDE, 2002a). O procedimento adequado para a predição dos valores genéticos utilizados na avaliação de plantas perenes tem sido o REML/BLUP (melhor predição linear não viesada) individual que consiste, basicamente, 
na predição de valores genéticos dos efeitos aleatórios do modelo estatístico, associado às observações fenotípicas, ajustando-se os dados aos efeitos fixos e ao número desigual de informações nas parcelas, por meio de metodologia de modelos mistos (HENDERSON et al., 1959).

Nesse contexto, o presente estudo estimou parâmetros genéticos e valores genéticos preditos pela metodologia de REML/BLUP para caracteres de crescimento, em um teste de progênies de irmãos completos aos 2, 5 e 7 anos de idade para selecionar genitores e indivíduos superiores de Eucalyptus em um programa de SRR no estado de Minas Gerais, Brasil.

\section{MATERIAL E MÉTODOS}

\section{Área experimental}

O estudo foi realizado em um teste de progênies de híbridos interespecíficos de irmãos completos, com 286 cruzamentos de polinização controlada em um pomar de semente clonal (PSC) de E. urophylla S. T. Blake, denominado (PSC 2000), no qual 33 árvores funcionaram como parentais femininos, pertencentes ao acervo de melhoramento genético florestal da Empresa Aperam Bioenergia Ltda. Os parentais masculinos (doadores de pólen) foram 64 árvores selecionadas em bancos clonais, testes clonais e pomares de sementes, com base nos seus rendimentos volumétricos e variabilidade genética de espécies e híbridos (Tabela 1).

Tabela 1. Relação e quantidade $(n)$ dos materiais genéticos utilizados para a hibridação inter-específica. Table 1. List of genetic materials used for inter-specific hybridization.

\begin{tabular}{lrlc}
\hline Feminino (Origem) & $\boldsymbol{n}$ & Masculino (Origem) & $\boldsymbol{n}$ \\
\hline E. urophylla (Timor) & 28 & E. grandis & 4 \\
E. urophylla (Flores) & 5 & E. saligna & 2 \\
Total & 33 & E. urophylla (Flores) & 2 \\
& E. urophylla (Timor) & 1 \\
& E. robusta & 1 \\
& E. resinífera & 5 \\
& Híbrido espontâneo: E. urophylla & 12 \\
& Híbrido E. urophylla x E. grandis & 4 \\
& Híbrido E. urophylla x (E. camaldulensis x E. grandis) & 5 \\
& Híbrido E. urophylla x [E. urophilla x (E. camaldulensis x E. grandis)] & 3 \\
& Híbrido E. camaldulensis x E. grandis & 6 \\
& Híbrido E. resinifera x E. grandis & 7 \\
& Híbrido E. urophylla x E. pellita & 1 \\
& Híbrido E. pellita x E. grandis & 2 \\
& Híbrido E. pellita x (E. grandis x E. tereticornis) & 1 \\
& Híbrido E. robusta x E. urophylla & 1 \\
& Híbrido E. robusta x E. grandis & 2 \\
& Híbrido E. saligna x E. botryoides & 4 \\
& Híbrido E. saligna x E. grandis & 1 \\
& Total & 64 \\
\hline
\end{tabular}

O experimento foi implantado em novembro de 2003, no município de Itamarandiba no Estado de Minas Gerais ( $17^{\circ} 44^{\prime} 45^{\prime \prime} \mathrm{S}, 42^{\circ} 45^{\prime} 11^{\prime \prime} \mathrm{W}$ e altitude $\left.1.000 \mathrm{~m}\right)$. A condição de clima e solo da região, onde se encontra o teste de híbridos, é de precipitação pluviométrica média anual de 1166 $\mathrm{mm}$, temperatura média de $21^{\circ} \mathrm{C}$ e o clima, conforme classificação de Köppen é tropical de altitude, temperado úmido com inverno seco e verão quente (Cwa). O solo é um latossolo vermelho distrófico típico e vermelho amarelo distrófico típico, com textura argilosa ou muito argilosa, bem estruturada. A topografia é plana (chapada) e a vegetação nativa é de Cerrado.

\section{Delineamento experimental e coleta de dados}

O delineamento experimental utilizado foi o de blocos casualizados, com oito repetições em parcelas lineares de seis plantas, para 286 tratamentos (cruzamentos) ou famílias. O espaçamento de plantio foi de $3 \mathrm{~m}$ entre linhas e de $2 \mathrm{~m}$ entre plantas na linha de plantio, perfazendo uma área contígua de 10 ha. Foram efetuadas medições nas árvores do experimento aos 2, 5 e 7 anos de idade das plantas, sendo a ultima na idade em que se faz a colheita da madeira para fabricação de carvão 
vegetal. Os caracteres medidos foram: Altura (ALT, m), utilizando-se clinômetro Suunto PM-5 360 PC; Diâmetro à altura do peito (DAP, $\mathrm{cm})$, utilizando-se fita métrica; sobrevivência (porcentagem). $\mathrm{O}$ volume individual das árvores foi calculado por, $V O L=\left(\pi d^{2} / 4\right) h 0,5$, em que $d$ e o DAP, $h$ e a ALT e 0,5 valor aplicado para fator de forma da árvore. O volume por hectare foi calculado por, $V O L=\left(\pi d^{2} / 4\right) h 0,5 * 1391$, em que 1391 e o numero de árvores sobreviventes por hectare no espaçamento de plantio $3 \times 2 \mathrm{~m}$.

\section{Análise estatística dos dados}

Estimativas de componentes de variâncias, parâmetros genéticos para caracteres de crescimento e a seleção individual ao nível de plantas foram obtidas pelo método de máxima verossimilhança restrita e melhor predição linear não viciada (REML/BLUP), empregando-se o software SELEGEN-REM/BLUP e o Modelo 88 deste software (RESENDE 2016). O modelo estatístico utilizado pode ser dado por:

$$
Y=X r+Z m+W f+T c+S p+e
$$

em que: y é o vetor de dados; r é o vetor dos efeitos de repetição (assumidos como fixos) somados à média geral; $\mathrm{m}$ é o vetor dos efeitos de genitores da população de machos (assumidos como aleatórios); f é o vetor dos efeitos de genitores da população de fêmeas (assumidos como aleatórios); c é o vetor dos efeitos da capacidade específica de combinação dos genitores da população de machos com os genitores da população de fêmeas (aleatórios); p: é o vetor dos efeitos de parcela (aleatórios); e: é o vetor de erros ou resíduos (aleatórios). As letras maiúsculas representam as matrizes de incidência para os referidos efeitos. As variáveis quantitativas e os parâmetros genéticos foram analisados conforme as equações abaixo admitindo que as progênies de polinização controlada sejam irmãos completos:

$\sigma_{g m}^{2}$ : estimativa da variância genética entre machos ou genitores da população $1 \mathrm{em}$ cruzamento com a população 2 , a qual estima $(1 / 4)$ da variação genética aditiva correspondente;

$\sigma_{g f}^{2}$ : estimativa da variância genética entre fêmeas ou genitores da população 2 em cruzamento com a população 1, a qual estima (1/4) da variação genética aditiva correspondente;

$\sigma_{a}^{2}=2\left(\sigma_{g m}^{2}+\sigma_{g f}^{2}\right)$ : estimativa da variância aditiva interpopulacional;

$\sigma_{c \in c}^{2}$ : estimativa da variância da capacidade específica de combinação interpopulacional entre dois genitores, a qual estima (1/4) da variação genética de dominância correspondente;

$\sigma_{\text {parc }}^{2}$ : estimativa da variância entre parcelas;

$\sigma_{\varepsilon}^{2}$ : estimativa da variância residual;

$\sigma_{f}^{2}=\sigma_{g m}^{2}+\sigma_{g f}^{2}+\sigma_{c e c}^{2}+\sigma_{p a r}^{2}+\sigma_{\theta}^{2}$ : estimativa da variância fenotípica interpopulacional;

$\sigma_{\text {fiom }}^{2}=4 \sigma_{c e c}^{2} / \sigma_{f}^{2}$ : estimativa da variância de dominância interpopulacional;

$h_{a m}^{2}=h_{a}^{2}=\sigma_{a}^{2} / \sigma_{f}^{2}$ : estimativa da herdabilidade individual no sentido restrito na população 1 , ou seja, dos efeitos aditivos interpopulacionais;

$h_{a f}^{2}$ : estimativa da herdabilidade individual no sentido restrito na população 2 , ou seja, dos efeitos aditivos interpopulacionais;

$C_{C \theta C}^{2}=\sigma_{c \theta c}^{2} / \sigma_{f}^{2}$ : estimativa do coeficiente de determinação dos efeitos da capacidade específica de combinação;

$h_{\text {diom }}^{2}=\sigma_{d i m}^{2} / \sigma_{f}^{2}$ : estimativa da herdabilidade individual dos efeitos interpopulacionais de dominância;

$h_{a}^{2}$ : estimativa da herdabilidade interpopulacional individual no sentido restrito, média para as duas populações;

$h_{g}^{2}=\sigma_{a}^{2}+\left(\sigma_{\text {diom }}^{2} / \sigma_{f}^{2}\right)$ : estimativa da herdabilidade interpopulacional individual no sentido amplo, ou seja, dos efeitos genotípicos totais;

$h_{a r o b}^{2}$ : estimativa da herdabilidade individual no sentido amplo para sobrevivência;

$C_{\text {parc }}^{2}=\sigma^{2} / \sigma_{f}^{2}$ : estimativa do coeficiente de determinação dos efeitos de parcela;

$A_{g m}=\sqrt{\frac{h^{2}}{h^{2}}}$ : acurácia geral de machos;

$A_{g f}=\sqrt{h_{g f}^{2}}:$ acurácia geral de fêmeas;

$A_{g c}=\sqrt{h_{a}^{2}}$ : acurácia geral de cruzamento;

$C V_{g p}(\%)=100 \sqrt{0,5} \sigma_{a}^{2} / m$ : coeficiente de variação genotípica entre progênies;

$C V_{\theta}(\%)=100 \sqrt{\sigma_{\theta}^{2} / m}$ : coeficiente de variação residual; 
$C V_{f}=C V_{g p}(\%) / C V_{\epsilon}(\%)$ : coeficiente de variação relativa;

$m$ : média do caráter;

gmd $=\sigma_{d}^{2} / \sigma_{a}^{2}+\sigma_{d}^{2}$ : grau médio de dominância.

\section{RESULTADOS E DISCUSSÃO}

\section{Variação, sobrevivência e produtividade}

Para todos os períodos e caracteres estudados, o teste da razão de verossimilhança (LRT) para efeitos genotípicos e de parcela apresentaram valores altamente significativos $(p<0,01)$. Isso indica que existe variabilidade genética e possibilidade para obtenção de ganhos com seleção (SEBBENN et al., 2008). Indica também que o ambiente no bloco não é homogêneo, mas como o coeficiente de determinação dos efeitos de parcela $\left(C_{p a r}^{2}\right.$ ) foi baixo para os caracteres e idades de avaliação (variando de 0,010 a 0,073), denotando que o delineamento experimental utilizado (blocos casualizados) garantiu o controle ambiental no teste de progênies, logo, que as estimativas dos parâmetros por análise de variância são confiáveis.

Tabela 2. Parâmetros genéticos para crescimento em altura (ALT), diâmetro à altura do peito (DAP) e volume (VOL), aos 2, 5 e 7 anos de idade, em teste de progênies de polinização controlada de Eucalyptus sp.

Table 2. Genetic parameters for height (ALT), diameter at breast height (DAP) and volume (VOL) at 2, 5 and 7 years of age in a controlled pollinated progeny test (full siblings) of Eucalyptus sp.

\begin{tabular}{|c|c|c|c|c|c|c|c|c|c|}
\hline & $\overline{\text { ALT2 }}$ & ALT5 & $\overline{\text { ALT7 }}$ & $\overline{\text { DAP2 }}$ & $\overline{\text { DAP5 }}$ & $\overline{\text { DAP7 }}$ & $\overline{\text { VOL2 }}$ & VOL5 & $\overline{\text { VOL7 }}$ \\
\hline $\operatorname{LRT}(\mathrm{G})$ & $49,7^{* *}$ & $70,4^{\star *}$ & $39,9^{* *}$ & $41,4^{\star *}$ & $87,1^{* *}$ & $60,8^{* *}$ & $47,2^{* \star}$ & $97,2^{* *}$ & $68,2^{* \star}$ \\
\hline $\mathrm{LRT}(\mathrm{P})$ & $66,0^{* *}$ & $44,5^{\star *}$ & $29,6^{* *}$ & $40,6^{\star *}$ & $34,3^{\star *}$ & $27,6^{\star *}$ & $46,9^{* \star}$ & $37,1^{* *}$ & $31,9^{\star *}$ \\
\hline$C_{p a r}^{2}$ & 0,071 & 0,073 & 0,039 & 0,018 & 0,017 & 0,010 & 0,031 & 0,018 & 0,014 \\
\hline$h_{a s o b *}^{2}$ & 0,22 & 0,19 & 0,04 & 0,22 & 0,19 & 0,04 & 0,22 & 0,19 & 0,04 \\
\hline$m$ & 10,10 & 22,07 & 24,83 & 8,04 & 14,41 & 15,263 & 0,026 & 0,188 & 0,260 \\
\hline$h_{a m}^{2}$ & $0,26 \pm 0,04$ & $0,41 \pm 0,03$ & $0,17 \pm 0,02$ & $0,23 \pm 0,03$ & $0,43 \pm 0,03$ & $0,27 \pm 0,03$ & $0,29 \pm 0,04$ & $0,53 \pm 0,04$ & $0,34 \pm 0,03$ \\
\hline$h_{a f}^{2}$ & 0,21 & 0,08 & 0,05 & 0,13 & 0,06 & 0,05 & 0,15 & 0,07 & 0,06 \\
\hline$C_{c e c}^{2}$ & 0,042 & 0,022 & 0,025 & 0,027 & 0,021 & 0,021 & 0,031 & 0,023 & 0,021 \\
\hline$h_{\text {diom }}^{2}$ & 0,17 & 0,09 & 0,10 & 0,11 & 0,09 & 0,09 & 0,12 & 0,09 & 0,09 \\
\hline$h_{a}^{2}$ & 0,23 & 0,25 & 0,11 & 0,18 & 0,25 & 0,16 & 0,22 & 0,30 & 0,20 \\
\hline gmd & 0,418 & 0,266 & 0,476 & 0,379 & 0,258 & 0,351 & 0,375 & 0,234 & 0,306 \\
\hline$H_{a}^{2}$ & 0,41 & 0,34 & 0,21 & 0,29 & 0,33 & 0,25 & 0,35 & 0,39 & 0,28 \\
\hline$A_{g m}$ & 0,763 & 0,885 & 0,788 & 0,786 & 0,900 & 0,858 & 0,807 & 0,9141 & 0,879 \\
\hline$A_{g f}$ & 0,829 & 0,795 & 0,732 & 0,803 & 0,771 & 0,746 & 0,815 & 0,7798 & 0,758 \\
\hline$A_{g c}$ & 0,853 & 0,874 & 0,841 & 0,820 & 0,892 & 0,867 & 0,840 & 0,905 & 0,878 \\
\hline$C V_{g p}(\%)$ & 4,5 & 3,7 & 5,7 & 5,3 & 6,2 & 8,5 & 13,5 & 16,5 & 20,6 \\
\hline$C V_{\theta}(\%)$ & 5,0 & 4,4 & 8,6 & 6,2 & 6,4 & 10,6 & 14,7 & 15,5 & 23,6 \\
\hline $\mathrm{CV}_{\tau}$ & 0,90 & 0,85 & 0,66 & 0,85 & 0,96 & 0,80 & 0,92 & 1,07 & 0,87 \\
\hline
\end{tabular}

LRT(G) e LRT(P) são o teste da razão de verossimilhança para genótipos e parcelas, respectivamente; ${ }^{*} * \mathrm{P}<0,0 \mathrm{0} ; C_{\text {. }}^{2}$ : coeficiente de determinação dos efeitos de parcelas; $h^{2}$ : herdabilidade individual no sentido amplo para sobrevivência; $m:$ média do caráter; $h_{\text {mp }}^{2}$ e $h_{\text {af são }}^{2}$ a herdabilidade individual no sentido restrito para os machos e fêmeass, respectivamente; \pm EP: erro padrão da media; $C^{2}$ : coeficiente de determinação dos efeitos da Capacidade Específica de Combinação; $h_{\text {jom }}^{2}$ : herdabilidade individual dos efeitos de dominância na população; $h_{a}^{2}$ : herdabilidade individual no sentido restrito para a população; $g m d^{\text {fom }}:$ grau médio de dominância; $H_{a}^{2}$ : herdabilidade individual no sentido amplo; $A_{g m}, A_{g f}$ e $A_{g c}$ são a acurácia geral de machos, fêmeas e geral de cruzamentos, respectivamente; $C V V_{g p}(\%), C V V_{e}(\%)$ e $C V$, são os coeficientes de variação genotípico entre progênies, residual e variação relativa, respectivamente; *Analisado pelo Modelo I 83 por se tratar de variável binomial.

A sobrevivência foi alta variando de $88,6 \%$ aos 2 anos de idades a $83,5 \%$ aos 7 anos de idade, indicando alta adaptação dos genótipos resultantes dos cruzamentos ao ambiente. A herdabilidade individual no sentido amplo para sobrevivência $\left(h_{a s o b}^{2}\right)$ decresceu com o aumento da idade $(0,22$ para 0,04), indicando que não há ação gênica sobre a sobrevivência (Tabela 2). Contudo, também 
indica que aos 7 anos de idade, o controle genético para sobrevivência é baixo e terá baixo efeito na seleção entre as progênies.

As progênies apresentaram aos 7 anos de idade média geral de ALT $(24,82 \mathrm{~m})$, DAP $(15,25 \mathrm{~cm})$, o que corresponde a um VOL individual de $0,26 \mathrm{~m}^{3}$ e a uma produtividade média em VOL para o teste ou todos os cruzamentos realizados de $51,64 \mathrm{~m}^{3}$. ha ${ }^{-1}$. nno $^{-1}$, aplicado um fator de forma de 0,5 para o cálculo de VOL (Tabela 2). Esta produtividade por hectare é aproximadamente 5,7 vezes maior do que a apresentada por plantios de Eucalyptus na região na década de $70\left(9,0 \mathrm{~m}^{3} \cdot \mathrm{ha}^{-1}\right.$. ano $\left.{ }^{-1}\right)$.

\section{Parâmetros genéticos}

A herdabilidade aditiva individual dos machos $\left(h_{\mathrm{am}}^{2}\right)$ para o caráter VOL variou de 0,29 aos 2 anos a 0,53 aos 5 anos e em seguida decresceu para 0,33 aos 7 anos de idade. Isto indica interação dos efeitos aditivos e ambientais, ao longo do período de crescimento das progênies em relação à população genitora masculina. Para a população feminina, os valores da herdabilidade individual no sentido restrito nas fêmeas $\left(h_{c f}^{2}\right)$, os valores são relativamente menores se comparados aos valores da população de machos, variando de 0,15 aos 2 anos para 0,06 aos 5 anos e decrescendo para 0,05 aos 7 anos de idade. Este resultado indica poucas possibilidades de ganhos genéticos advindos das mães (RESENDE, 2002b).

O coeficiente de determinação dos efeitos da capacidade específica de combinação $\left(C_{c \in c}^{2}\right)$ está relacionado aos efeitos de dominância nos cruzamentos (RESENDE, 2007). Os valores para este coeficiente foram relativamente baixos, em torno de 0,02 para todas as idades e caracteres estudados. A herdabilidade individual dos efeitos de dominância da população segregante $\left(h_{\text {coom }}^{2}\right)$ apresentou valores baixos para todas as idades e caracteres (variando de 0,09 a 0,17), conforme Resende et al. (1995), resultado que corrobora com os valores do $C_{c \in c}^{2}$. Ressalta-se ainda que desvios de dominância são importantes na adaptação a variações ambientais como estresses biótico e abiótico.

A herdabilidade no sentido restrito em nível de plantas $\left(h_{a}^{2}\right)$ variou entre os caracteres e idades de baixos $(0,11)$ a médios $(0,30)$, foram menores na idade de 7 anos e foram maiores para o VOL. Esta herdabilidade indica variabilidade genética que é herdável na população ou a quantidade relativa de variância genética útil para o melhoramento da descendência por propagação sexual (FALCONER, 1987). No presente estudo, a $h_{a}^{2}$ indica as boas perspectivas em relação ao ganho genético dos caracteres de crescimento. A variação observada entre idades e caracteres é coerente com os demais estudos com espécies de Eucalyptus spp para os mesmos caracteres e idades, (BERG et al., 2017; GARCIA; NOGUEIRA, 2005; ROCHA et al., 2007).

O coeficiente de variação genotípica entre progênies $\left(C V_{g p}\right)$ foi baixo para os caracteres ALT e DAP (variando entre 3,7 a 8,5\%) e medianos para VOL (13,5 a 20,6\%), indicando o VOL como o caráter mais apropriado para seleção. Neste caso, o genótipo desempenha maior importância na expressão do fenótipo que o ambiente devido ao fato de que caracteres de ação poligênica como o volume possuem interações gênicas complexas, são altamente influenciados pelo ambiente, dificultando a identificação dos genótipos superiores com base no fenótipo (BESPALHOK et al., 2007a). Daí a importância de se conhecer o valor da ação gênica que predomina na formação dos novos genótipos, se aditiva, de dominância e epistática. Somente os efeitos aditivos conferem segurança aos ganhos preditos no programa de melhoramento (BESPALHOK et al., 2007b).

O grau médio de dominância ( $g$ md ) apresentou valores entre 0,35 para VOL a 0,47 para ALT ambos aos 7 anos de idade das plantas. Estes valores indicam que os locos em heterozigose estão contribuindo consideravelmente para a maior capacidade de resposta às variações ambientais (RESENDE, 2002b). Contudo, na fase crítica de estabelecimento das plantas, que ocorre aos dois primeiros anos de vida, quando os genótipos deixam as condições favoráveis do viveiro, com controle de irrigação, fertilização e manejo de insolação, para a condição extremamente inóspita do campo, os fatores genéticos aditivos e de dominância são mais pronunciados. A partir desta fase, com as plantas já estabelecidas, os fatores ambientais são mais determinantes e o sucesso do empreendimento é em grande parte dependente do manejo do ambiente (tratos culturais: mato competição, adubação, controle de pragas e doenças).

A herdabilidade individual entre os sexos no sentido amplo $\left(H_{a}^{2}\right)$ foi mediana, variando entre caracteres e idades de 0,21 a 0,41, foi menor em todos os caracteres na idade de 7 anos. Estes valores indicam a possibilidade de sucesso no aumento da produtividade por seleção dentro da população. 
As acurácias de machos $\left(A_{g m}\right)$, de fêmeas $\left(A_{g f}\right)$ e dos cruzamentos $\left(A_{g c}\right)$ foram relativamente altas $(>0,75)$, conforme classificação de Resende et al. (1995). Isto indica que a seleção a ser realizada terá garantia de acerto acima de $70 \%$ e reforça que o desenho experimental foi eficiente para garantir o controle dos efeitos ambientais (NUNES et al., 2016; RESENDE; DUARTE, 2007).

$\mathrm{O}$ coeficiente de variação experimental $\left(\mathrm{CV}_{\epsilon}\right)$ foi similar, embora geralmente maior do que o $C V_{g p}$. Isso resultou em um coeficiente de variação relativa $\left(C V_{+}\right)$em geral maior do que 0,85 , variando de 0.65 para ALT7 $(0,65)$ a 1,07 para VOL. Como valores próximos de unidade (1) indicam forte relação entre efeitos genotípicos e ambientais (MAIA et al., 2009), logo existe alta probabilidade de obter-se ganhos genéticos com a seleção. Os resultados deste estudo confirmam que o material testado tem alto potencial para ser melhorado pela seleção.

\section{Seleção dos materiais genéticos}

A partir da classificação dos cruzamentos (Tabela 3), com base no valor genético aditivo e no ponto de corte do VOL de $65 \mathrm{~m}^{3}$.ha- $\mathrm{hano}^{-1}$ foi efetuada a seleção dos 32 melhores cruzamentos, ou seja, famílias com maior produtividade volumétrica. Este volume de corte representa um aumento na produtividade volumétrica por hectare de aproximadamente 7,2 vezes maior do que a apresentada por plantios de Eucalyptus na região na década de $70\left(9,0 \mathrm{~m}^{3} \cdot \mathrm{ha}^{-1}\right.$.ano $\left.{ }^{-1}\right)$ e 1,3 vezes maior que o do teste $\left(51,64 \mathrm{~m}^{3} \cdot \mathrm{ha}^{-1} \cdot\right.$ ano $\left.^{-1}\right)$. Contudo, para aumentar fortemente a produtividade recomenda-se três estratégias de seleção: i) estabelecer um pomar de recombinação por polinização aberta, composto de 256 indivíduos, sendo oito por cruzamento (o melhor de cada repetição) cujo rendimento médio é de $74,24 \mathrm{~m}^{3} \cdot \mathrm{ha}^{-1}$.ano ${ }^{-1}$; ii) estabelecer um pomar de hibridação por polinização controlada, composto de 65 indivíduos, sendo um por cruzamento (o melhor) totalizando 32 filhos, mais os 16 melhores genitores femininos e mais os 17 genitores masculinos, com produtividade média também de 74,24 $\mathrm{m}^{3}$.ha ${ }^{-1}$.ano ${ }^{-1}$; no intuito de se dar prosseguimento aos trabalhos de melhoria contínua da produtividade pela Seleção Recorrente Recíproca (SRR) a médio e longo prazos e, iii) Com base na variância genética total, a partir da classificação das progênies sugere-se selecionar os 10 melhores indivíduos para clonagem (Tabela 4), objetivando ganhos a curto prazo. Esta população tem rendimento médio de $86,08 \mathrm{~m}^{3} \cdot \mathrm{ha}^{-1} \cdot \mathrm{ano}^{-1}$. Para as estratégias de seleção I e II, o aumento da produtividade foi 1,4 vezes e para a situação III foi 1,7 maior que o do teste $\left(51,64 \mathrm{~m}^{3} \cdot \mathrm{ha}^{-1}\right.$.ano $\left.{ }^{-1}\right)$.

Tabela 3. Seleção dos cruzamentos (Cruz) que produziram rendimentos florestais iguais ou superiores a $65 \mathrm{~m}^{3} \cdot \mathrm{ha}^{-1}$. ano ${ }^{-1}$ aos sete anos de idade.

Table 3. Selection of crosses that produced forest yields equal to or greater than $65 \mathrm{~m}^{3}$.ha-1.year-1 at seven years of age.

\begin{tabular}{|c|c|c|c|c|c|c|c|}
\hline \multirow{2}{*}{$\begin{array}{l}\text { Classi- } \\
\text { ficação }\end{array}$} & \multicolumn{3}{|c|}{ Crescimento $\left(\mathrm{m}^{3}\right)$} & \multicolumn{2}{|c|}{ Genitor feminino } & \multicolumn{2}{|r|}{ Genitor masculino } \\
\hline & Cruz & Individual & halano & Nome & Especie & Nome & Especie \\
\hline$\overline{1}$ & 278 & 0,4219 & 83,29 & 3.026 & E. urophylla (Timor) & Gran006 & E. grandis \\
\hline 2 & 117 & 0,4061 & 80,17 & 2.044 & E. urophylla (Timor) & Sal784 & E. saligna \\
\hline 3 & 428 & 0,4008 & 79,13 & 3.052 & E. urophylla (Timor) & Gran045 & E. grandis \\
\hline 4 & 420 & 0,3976 & 78,49 & 3.052 & E. urophylla (Timor) & Gran004 & E. grandis \\
\hline 5 & 251 & 0,3956 & 78,10 & 3.026 & E. urophylla (Timor) & Gran005 & E. grandis \\
\hline 6 & 132 & 0,3937 & 77,72 & 2.044 & E. urophylla (Timor) & Salxbot098 & E. saligna $\times$ E. botryoides \\
\hline 7 & 62 & 0,3913 & 77,25 & 1.294 & E. urophylla (Timor) & Gran004 & E. grandis \\
\hline 8 & 292 & 0,3880 & 76,60 & 3.026 & E. urophylla (Timor) & Salxbot098 & E. saligna $\times$ E. botryoides \\
\hline 9 & 320 & 0,3852 & 76,05 & 3.336 & E. urophylla (Timor) & Gran004 & E. grandis \\
\hline 10 & 274 & 0,3810 & 75,22 & 3.026 & E. urophylla (Timor) & Salxbot112 & E. saligna $x$ E. botryoides \\
\hline 11 & 280 & 0,3775 & 74,53 & 3.026 & E. urophylla (Timor) & Gran045 & E. grandis \\
\hline 12 & 131 & 0,3744 & 73,91 & 2.044 & E. urophylla (Timor) & Salxbot112 & E. saligna $\times$ E. botryoides \\
\hline 13 & 325 & 0,3712 & 73,28 & 3.336 & E. urophylla (Timor) & Gran045 & E. grandis \\
\hline 14 & 393 & 0,3675 & 72,55 & 3.351 & E. urophylla (Timor) & AEC0099 & Hib. esp. E. urophylla \\
\hline 15 & 293 & 0,3639 & 71,84 & 3.025 & E. urophylla (Timor) & Salxbot784 & E. saligna $x$ E. botryoides \\
\hline 16 & 202 & 0,3608 & 71,23 & 3.024 & E. urophylla (Timor) & Gran004 & E. grandis \\
\hline 17 & 410 & 0,3579 & 70,66 & 3.052 & E. urophylla (Timor) & 4.206 & E. urophylla (Flores) \\
\hline 18 & 431 & 0,3552 & 70,12 & 3.052 & E. urophylla (Timor) & AEC0760 & E. camaldulensis $\times$ E. grandis \\
\hline 19 & 358 & 0,3524 & 69,57 & 3.046 & E. urophylla (Timor) & AEC0099 & Hib. esp. E. urophylla \\
\hline 20 & 396 & 0,3499 & 69,08 & 3.512 & E. urophylla (Timor) & AEC0099 & Hib. esp. E. urophylla \\
\hline 21 & 491 & 0,3476 & 68,62 & 4.206 & E. urophylla (Flores) & Gran106 & E. grandis \\
\hline 22 & 504 & 0,3455 & 68,21 & 4.254 & E. urophylla (Flores) & Gran045 & E. grandis \\
\hline 23 & 30 & 0,3435 & 67,81 & 1.254 & E. urophylla (Timor) & AEC0099 & Hib. esp. E. urophylla \\
\hline 24 & 237 & 0,3417 & 67,46 & 3.025 & E. urophylla (Timor) & AEC1559 & E. uro x $[$ E. uro (E. cam x E. gran)] \\
\hline
\end{tabular}

Sci. For., Piracicaba, v. 46, n. 119, p. 405-413, set. 2018 DOI: dx.doi.org/10.18671/scifor.v46n119.08 
Henriques et al. - Seleção genética em teste de progênies de irmãos

completos de Eucalyptus para a produção de carvão vegetal

Tabela 3 - Continuação. Seleção dos cruzamentos (Cruz) que produziram rendimentos florestais iguais ou superiores a $65 \mathrm{~m}^{3}$. ha- ${ }^{-1}$. ano ${ }^{-1}$ aos sete anos de idade.

Table 3 - Continuation. Selection of crosses that produced forest yields equal to or greater than $65 \mathrm{~m}^{3} \cdot \mathrm{ha}^{-1} \cdot \mathrm{year}^{-1}$ at seven years of age.

\begin{tabular}{lccc|cl|c}
\hline Classi- & \multicolumn{3}{|c|}{ Crescimento $\left(\mathbf{m}^{\mathbf{3}}\right)$} & \multicolumn{2}{c}{ Genitor feminino } & Genitor masculino \\
ficação & Cruz & Individual & ha/ano & Nome & Especie & Nome Especie \\
\hline 25 & 317 & 0,3400 & 67,12 & 3.336 & E. urophylla (Timor) & 4.206 E. urophylla (Flores) \\
26 & 447 & 0,3384 & 66,81 & 4.199 & E. urophylla (Flores) & AEC1513 E. uro $x$ [ E. uro (E. cam x E. gran)] \\
27 & 47 & 0,3369 & 66,51 & 1.294 & E. urophylla (Timor) & AEC1633 E. pellita x E. grandis \\
28 & 36 & 0,3355 & 66,23 & 1.254 & E. urophylla (Timor) & AEC0060 Hib. esp. E. urophylla \\
29 & 28 & 0,3341 & 65,96 & 1.254 & E. urophylla (Timor) & AEC0258 Hib. esp. E. urophylla \\
30 & 9 & 0,3327 & 65,68 & 1.034 & E. urophylla (Timor) & AEC1633 E. pellita x E. grandis \\
31 & 490 & 0,3314 & 65,42 & 4.026 & E. urophylla (Flores) & AEC0060 Hib. esp. E. urophylla \\
32 & 416 & 0,3302 & 65,19 & 3.052 & E. urophylla (Timor) & AEC1513 E. uro x E. uro (E. cam $x$ E. gran)] \\
\hline
\end{tabular}

São 32 cruzamentos onde participaram 16 genitores femininos e 17 genitores masculinos.

Tabela 4. Relação dos indivíduos a serem clonados.

Table 4. Relation of individuals to be cloned.

\begin{tabular}{llllcc}
\hline \multirow{2}{*}{ Ranking } & Cruzamento & Bloco & Árvore & \multicolumn{2}{c}{ Crescimento $\left(\mathbf{m}^{\mathbf{3}}\right)$} \\
\hline 1 & 420 & 5 & 4 & Individual & Por ha/ano \\
2 & 278 & 5 & 1 & 0,4436 & 87,63 \\
3 & 251 & 5 & 6 & 0,4429 & 87,49 \\
4 & 3 & 1 & 0,4395 & 86,82 \\
5 & 278 & 3 & 5 & 0,4378 & 86,48 \\
6 & 132 & 8 & 1 & 0,4360 & 86,13 \\
7 & 117 & 2 & 1 & 0,4343 & 85,79 \\
8 & 292 & 6 & 4 & 0,4328 & 85,49 \\
9 & 278 & 8 & 1 & 0,4314 & 85,22 \\
10 & 420 & 7 & & 0,4303 & 85,00 \\
\hline Média & & & & 0,4294 & 84,82 \\
\hline
\end{tabular}

\section{CONCLUSÕES}

a) As famílias de híbridos interespecíficos resultantes dos cruzamentos realizados apresentam variabilidade genética para todos os caracteres e idades avaliadas;

b) As estratégias de seleção propostas predizem altos ganhos genéticos e o aumento da produtividade dos plantios da região estudada.

\section{REFERÊNCIAS BIBLIOGRÁFICAS}

ASSIS, T. F. Melhoramento genético do eucalipto. Informe Agropecuário, Belo Horizonte, v. 18, n. 185, p. 32-51, 1996.

ASSIS, T. F.; HENRIQUES, E. P.; FERNANDES, J. C. Relatório técnico descritivo para obtenção do registro nacional dos cultivares: relatório n. 1: espécies puras. Itamarandiba: Arcelor Mittal BioEneria, 2007. 11 p.

BERG, G. D.; VERRYN, S. D.; CHIRWA, P. W.; DEVENTER, F. Realised genetic gains and estimated genetic parameters of two Eucalyptus grandis $\times$ E. urophylla hybrid breeding strategies. Southern Forests, Pretoria, p.111, 2017. Doi: 10.2989/20702620.2016.1263010.

BESPALHOK F. J. C.; GUERRA, E. P.; OLIVEIRA, R. Noções de Genética Quantitativa. In: BESPALHOK F. J. C.; GUERRA, E. P.; OLIVEIRA, R. Melhoramento de Plantas. Curitiba: FUPEF, 2007a.

BESPALHOK F. J. C.; GUERRA, E. P.; OLIVEIRA, R. Introdução ao Melhoramento de Plantas. In: BESPALHOK F. J. C.; GUERRA, E. P.; OLIVEIRA, R. Melhoramento de Plantas. Curitiba: FUPEF, 2007b.

BOUDET, A. M. A. New view of lignification. Trends in Plant Science, v. 3, p. 67-71, 1998. 
CRUZ, C. D.; CARNEIRO, P. C. S. Modelos biométricos aplicados ao melhoramento genético. Viçosa: Universidade Federal de Viçosa, 2003. v.2. 585 p.

FALCONER, D. S. Introdução à genética quantitativa. Viçosa: Universidade Federal de Viçosa, Imprensa Universitária, 1987. 279 p.

GARCIA, C. H.; NOGUEIRA, M. C. S. N. Utilização da metodologia REML/BLUP na seleção de clones de eucalipto. Scientia Forestalis, Piracicaba, n. 68, p. 107-112, 2005.

GOLFARI, L. Zoneamento ecológico do Estado de Minas Gerais para reflorestamento. Belo Horizonte: PNUD/FAO/IBDF, 1975. 65 p. (Série Técnica BRA/71/545).

HENDERSON, C. R.; KEMPTHORNE, O.; SEARLE, S. R.; KROSIGKVON, C. M. The estimation of environmental and genetic trends from records subject to culling. Biometrics, v.15, n. 6, p. 192-218, 1959.

IBÁ - INDÚSTRIA BRASILEIRA DE ÁRVORES. Relatório anual: ano base 2015. Brasília: IBA, 2016. 96 p.

MAIA, M. C. C.; RESENDE, M. D. V.; PAIVA, J. R.; CAVALCANTI, J. J. V.; BARROS, L. M.. Seleção simultânea para produção, adaptabilidade e estabilidade genotípicas em clones de cajueiro, via modelos mistos. Pesquisa Agropecuária Tropical, Goiânica, v. 39, n. 1, p.43-50, 2009.

NUNES, A. C. P, SANTOS, G. A, RESENDE, M. D. V, SILVA, L. D, HIGA, A ASSIS, T. F. Estabelecimento de zonas de melhoramento para clones de eucalipto no Rio Grande do Sul. Scientia Forestalis, Piracicaba, v.44, n.111, p. 563-574, 2016.

RESENDE, M. D. V. Software Selegen-REML/BLUP: a useful tool for plant breeding. Crop Breeding and Applied Biotechnology, Viçosa, v.16, p.330-339, 2016.

RESENDE, M. D. V. Matemática e estatística na análise de experimentos e no melhoramento genético. Colombo: Embrapa Florestas, 2007. 562 p

RESENDE, M. D. V. SELEGEN-REML/BLUP - Seleção genética computadorizada: manual do usuário. Colombo: Embrapa - CNPF, 2002a b. 67p.

RESENDE, M. D. V. Genética Biométrica e Estatística no Melhoramento de Plantas Perenes. Brasília: Embrapa informações tecnológicas, 2002b. 975 p.

RESENDE, M. D. V.; DUARTE, J. B. Precisão e controle de qualidade em experimentos de avaliação de cultivares. Pesquisa Agropecuária Tropical, Goiânia, v. 37, p. 182-194, 2007.

RESENDE, M. D. V.; HIGA, A. R. Estratégias de melhoramento para eucaliptos visando a seleção de híbridos. Boletim de Pesquisa Florestal, Colombo, n. 21, p. 49-60, 1990.

RESENDE, M. D. V.; ARAÚJO, A. J.; SAMPAIO, P. T. B.; WIECHETECK, M. S. S. Accuracy, confidence ranges and variance of response associated to 22 selection methods in Pinus caribaea var. hondurensis. Revista Floresta, Curitiba, v. 24, p. 34-45, 1995.

ROCHA, M. G. B.; PIRES, I. E.; ROCHA, R. B.; XAVIER, A.; CRUZ, C. D. Seleção de genitores de Eucalyptus grandis e de Eucalyptus urophylla para produção de híbridos interespecíficos utilizando REML/ BLUP e informação de divergência genética. Revista Árvore, Viçosa, v. 31, n. 6, p. 977-987, 2007.

SEBBENN, A. M.; VILAS BOAS, O.; MAX, J. C. M. Altas herdabilidades e ganhos na seleção para caracteres de crescimento em teste de progenies de polinização aberta de Pinus elliottii Engelm var. elliottii aos 25 anos de idade em Assis -SP. Revista do Instituto Florestal, São Paulo, v. 20, n. 2, p. 95-102, 2008.

Recebido em 02/05/2017

Aceito em 18/01/2018

Sci. For., Piracicaba, v. 46, n. 119, p. 405-413, set. 2018 DOI: dx.doi.org/10.18671/scifor.v46n119.08 
